\title{
Densidade de incidência de infecção primária de corrente sanguínea associada ao cateter venoso central no Brasil
}

Incidence density of primary bloodstream infection associated with central venous catheter in Brazil

Densidad de incidencia de la infección primaria del torrente sanguíneo asociada al catéter venoso central en Brasil

Recebido: 06/08/2021 | Revisado: 13/08/2021 | Aceito: 16/08/2021 | Publicado: 19/08/2021

\author{
Maria Renata da Silva Galvão \\ ORCID: https://orcid.org/0000-0003-2880-8362 \\ Universidade Potiguar, Brasil \\ E-mail: renata-rn@hotmail.com \\ Mayara Cavalcante Rodrigues \\ ORCID: https://orcid.org/0000-0003-4979-318X \\ Universidade Potiguar, Brasil \\ E-mail: mayara10036@gmail.com \\ Renata Kely Pimentel da Cunha \\ ORCID: https://orcid.org/0000-0003-4533-5851 \\ Universidade Potiguar, Brasil \\ E-mail: rekely@hotmail.com \\ Vera Lúcia Morais da Silva \\ ORCID: https://orcid.org/0000-0002-7675-1199 \\ Universidade Potiguar, Brasil \\ E-mail: veraleite035@gmail.com \\ Camila Priscila Abdias do Nascimento \\ ORCID: https://orcid.org/0000-0002-0245-2663 \\ Universidade Potiguar, Brasil \\ E-mail: camilapriabd@gmail.com
}

\begin{abstract}
Resumo
Introdução: As Infecções Relacionadas à Assistência à Saúde são aquelas cuja aquisição está relacionada ao cuidado em saúde e que não estavam presentes no momento em que precede a assistência, oriundas da interação entre profissional e paciente. Dentre as principais infecções adquiridas relacionadas ao cuidado na assistência à saúde, destaca-se a Infecção de Corrente Sanguínea, associada ao cateter venoso central. Objetivo: Evidenciar a densidade de incidência de infecção primária de corrente sanguínea relacionada ao cateter venoso central e fatores associados no período de 2015 e 2019 nas Unidades de Terapia Intensiva (UTI) adulta, pediátrica e neonatal no Brasil. Metodologia: Trata-se de um estudo exploratório e descritivo com abordagem quantitativa, por meio de levantamento de dados em fontes secundárias, no período de análise, nas plataformas da ANVISA e DATASUS. Resultado e Discussão: Consoante os dados coletados, o Brasil realizou um número crescente de notificações de Infecções Primárias de Corrente Sanguínea com Confirmação Laboratorial por ano, assim como, houve aumento no número de serviços de saúde que realizaram a notificação desse tipo de evento. Constatou-se, ainda, o decréscimo na densidade de incidência dessas infecções em UTI adulto de 0,89 e pediátrica de 1,35. Entretanto, na UTI neonatal ocorreu o acréscimo de 0,1. Conclusão: Observouse a redução na densidade da incidência de infecções e, também, incongruência na emissão desses dados, tanto na frequência quanto na quantidade de eventos. Essas notificações significam muito quando considerado a segurança do paciente, pois possibilitam estabelecer estratégias e metas que visam reduzir esses incidentes.
\end{abstract}

Palavra-chave: Assistência de enfermagem; Cateteres venosos centrais; Infecções relacionadas a cateter; Segurança do paciente.

\begin{abstract}
Introduction: Health Care-Related Infections (HCAIs) are infections acquired during health care and which were not present at the time when the care was provided, resulting from the interaction between professional and patient. Among the main health care-related infections acquired during health care, central venous catheter-associated bloodstream infection stands out. Objective: To evidence the incidence density of primary central venous catheter-related bloodstream infection and associated factors in the period 2015 and 2019 in adult, pediatric, and neonatal Intensive Care Units (ICUs) in Brazil. Methodology: This is an exploratory and descriptive study with a quantitative approach, through data collection from secondary sources, in the period of analysis, in ANVISA and DATASUS platforms. Results and Discussion: According to the data collected, Brazil had an increasing number of notifications of Primary Bloodstream
\end{abstract}


Infections with Laboratory Confirmation per year, as well as, there was an increase in the number of health services that performed the notification of this type of event. There was also a decrease in the incidence density of these infections in adult ICU of 0.89 and pediatric of 1.35 . However, in the neonatal ICU there was an increase of 0.1. Conclusion: A reduction in the density of infection incidence was observed, as well as incongruence in the emission of these data, both in frequency and quantity of events. These notifications mean a lot when considering patient safety, because they make it possible to establish strategies and goals aimed at reducing these incidents.

Keywords: Nursing care; Central venous catheters; Catheter-related infections; Patient safety.

\section{Resumen}

Introducción: Las infecciones relacionadas con la atención sanitaria (IRAS) son aquellas que se adquieren a través de la atención sanitaria y que no estaban presentes en el momento en que se prestó la atención, resultando de la interacción entre el profesional y el paciente. Entre las principales infecciones adquiridas relacionadas con la asistencia sanitaria, destaca la infección del torrente sanguíneo, asociada a los catéteres venosos centrales. Objetivo: Evidenciar la densidad de incidencia de la infección primaria de la corriente sanguínea relacionada con el catéter venoso central y los factores asociados en el período de 2015 a 2019 en las Unidades de Terapia Intensiva (UTI) adultas, pediátricas y neonatales en Brasil. Metodología: Se trata de un estudio exploratorio y descriptivo con enfoque cuantitativo, a través de la recolección de datos de fuentes secundarias, en el período de análisis, en las plataformas ANVISA y DATASUS. Resultados y Discusión: De acuerdo con los datos recogidos, Brasil tuvo un número creciente de notificaciones de infecciones primarias del torrente sanguíneo con confirmación de laboratorio por año, así como, hubo un aumento en el número de servicios de salud que realizaron la notificación de este tipo de evento. También se observó una disminución de la densidad de incidencia de estas infecciones en la UCI de adultos de 0,89 y en la pediátrica de 1,35. Sin embargo, en la UCI neonatal hubo un aumento de 0,1. Conclusión: Se observó una reducción en la densidad de la incidencia de las infecciones, así como una incongruencia en la emisión de estos datos, tanto en la frecuencia como en la cantidad de eventos. Estas notificaciones tienen un gran significado cuando se considera la seguridad del paciente, ya que permiten establecer estrategias y objetivos para reducir estos incidentes.

Palabras clave: Cuidados de enfermería; Catéteres venosos centrales; Infecciones relacionadas con el catéter; Seguridad del paciente.

\section{Introdução}

As Infecções Relacionadas à Assistência à Saúde (IRAS) são aquelas cuja aquisição está relacionada ao cuidado em saúde e que não estavam presentes no momento em que precede a assistência, oriundas da interação entre profissional e paciente, além de serem uma das prioridades do desafio global criado pela Organização Mundial de Saúde (OMS). A OMS, em 2004, apresentou o programa World Alliance for Patient Safety (Aliança Mundial para a Segurança do Paciente), em que foram destacadas as pesquisas que motivaram a criação da Classificação Internacional de Segurança do Paciente com conceitos e ações para mitigar eventos adversos resultantes do cuidado em saúde.

Nesse contexto, o Ministério da Saúde (MS) instituiu em 2013, no Brasil, o Programa Nacional de Segurança do Paciente, cujo objetivo é contribuir para a qualificação do cuidado e desenvolver estratégias para que todos os estabelecimentos de saúde possam reduzir os danos associados à assistência. Dentre os cuidados na assistência à saúde, destacam-se aqueles relacionados aos Cateteres Venosos Centrais (CVCs), que são dispositivos capazes de conduzir grande variedade de soluções. Entretanto, existe o risco de surgirem algumas complicações associadas, como a Infecção de Corrente Sanguínea (ICS), proveniente de causas multifatoriais e que apresenta aspectos e tratamentos distintos.

Cabe ressaltar que os principais fatores que contribuem para as Infecções de Corrente Sanguínea Relacionada ao Cateter (ICSRC) são advindos da colonização extraluminal e intraluminal, desencadeadas por bactérias que formam um biofilme no decorrer do tempo de permanência e conforme frequência de manipulação dos lúmens e hub. Além disso, a prática inadequada de preparo e administração de infusões, pode resultar em contaminação dessas soluções. Menos comumente, a disseminação hematogênica, que é a colonização da ponta do dispositivo.

Nessa perspectiva, as Infecções Primárias da Corrente Sanguínea (IPCS) podem apresentar desfechos sistêmicos graves, isto é, a bacteremia e consequentemente, a sepse, sem foco primário identificável (ANVISA, 2013). Haja visto que, de janeiro a maio de 2021, a septicemia alcançou uma taxa de mortalidade de 46,8 a cada 100 casos no Brasil, com um total de 21.036 óbitos 
em hospitais do país, de acordo com levantamento do Sistema de Informação de Saúde (Brasil, 2020), vislumbra-se a amplitude desse agravo. Para viabilizar o controle epidemiológico da ICS, utilizam-se subdivisões de acordo com critérios estabelecidos, para as principais variáveis, como IPCS laboratoriais e clínicas (ANVISA, 2013).

Sabe-se que essas infecções, quando associadas ao CVC, são responsáveis por cerca de $20 \%$ desse tipo de ocorrência nos hospitais da Europa e têm uma taxa de mortalidade em torno de 30\% (Karapanau et al., 2020). Analogamente, nos Estados Unidos, por ano, cerca de 250.000 dessas ocorrências são notificadas, com uma taxa de mortalidade aproximada de $25 \%$ e custo de tratamento que pode atingir o montante de até 60 mil dólares por evento (Chaiyakulsil \& Pharadornuwat, 2020).

Entende-se que, grande parte das ocorrências de infecções podem ser evitadas ao cumprir-se as medidas preventivas e implementá-las desde a inserção do CVC, como também, durante sua manutenção, manuseio e retirada. Diante do exposto, objetivou-se, neste estudo, evidenciar a densidade de incidência (DI) de infecção primária de corrente sanguínea relacionada ao CVC e fatores associados no período de 2015 a 2019 nas Unidades de Terapia Intensiva (UTI) adulta, pediátrica e neonatal no Brasil.

\section{Metodologia}

Trata-se de um estudo exploratório e descritivo com abordagem quantitativa. Segundo Gil (1999), a pesquisa exploratória visa modificar e esclarecer conceitos, ideias e possibilita uma visão abrangente e aproximativa sobre determinada temática estudada. Em contrapartida, conceitua a pesquisa descritiva como aquela cujo objetivo principal é a descrição das características de determinada população ou fenômeno estudado e relacionar variáveis com técnicas estabelecidas. Logo, optouse neste estudo pelo levantamento de informações em fontes secundárias.

A coleta dos dados referentes a densidade de incidência (DI) no período de 2015 a 2019, contou com as informações disponibilizadas no site da Agência Nacional de Vigilância Sanitária (ANVISA), no documento intitulado Boletim Segurança do Paciente e Qualidade em Serviços de Saúde no Brasil n²2 - Avaliação dos Indicadores Nacionais de Infecções Relacionadas à Assistência à Saúde (IRAS) e Resistência Microbiana (RM), Ano 2019. Ainda, de forma complementar, com o objetivo de abranger a temática e relacioná-la aos fatores inerentes à DI encontrada, como também, seguir os passos do estudo exploratório e descritivo, foi levantado neste Boletim, o quantitativo de infecção de corrente sanguínea associada a inserção de CVC notificadas no Brasil, no mesmo recorte temporal.

Realizou-se, também, a pesquisa no Sistema de Informação da Saúde (SIS), na plataforma do departamento de informática do Sistema Único de Saúde do Brasil (DATASUS), com vistas ao levantamento de dados sobre a quantidade total de autorização de inserção de cateter de longa permanência semi ou totalmente implantado, assim como a média de dias de internação para realização do procedimento pelo Sistema Único de Saúde (SUS). A pesquisa em ambas as plataformas utilizadas neste estudo ocorreu durante os meses de março a julho de 2021 e coletou os dados mais atualizados disponíveis. Outrossim, o embasamento teórico foi realizado através de consulta de artigos na Biblioteca Virtual de Saúde (BVS), Scielo Brasil e no repositório da ProQualis.

\section{Resultados e Discussão}

Durante os anos de 2015 a 2019 o Brasil realizou em média o implante de 33,62 cateteres de longa permanência semi ou totalmente implantado por dia, o que implica em torno de 12.238 cateteres implantados ao ano (BRASIL, 2020). Ao ser considerada a quantidade de procedimentos autorizados, a Região Sudeste (SE) foi responsável pela maior parte dos procedimentos, seguida das Regiões Sul (S), Nordeste (NE), Centro-Oeste $(\mathrm{CO})$ e Norte $(\mathrm{N})$, respectivamente, conforme o gráfico 1. Esses dados projetam a quantidade de cateteres a serem manipulados por equipes multiprofissionais em unidades de 
atendimento em saúde, clínicas ou hospitalares, de diferentes intensidades de cuidado, inclusive em UTI.

\title{
Gráfico 1: Média de implante de Cateteres semi ou totalmente implantados por ano de 2015 a 2019, de acordo com Região, para todas as faixas etárias
}

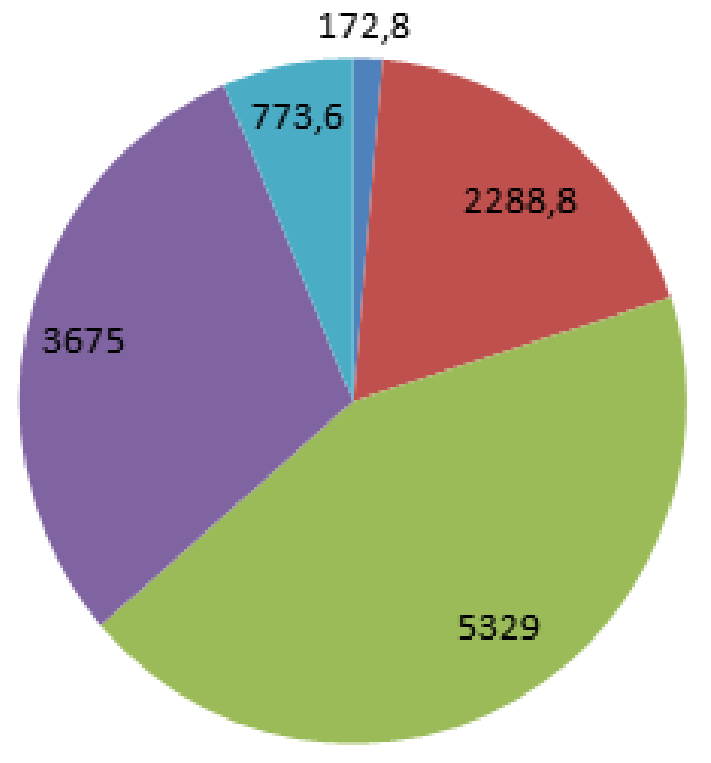

\author{
Norte \\ Nordeste \\ Sudeste \\ n Sul \\ Centro-Oeste
}

Fonte: Autores, com dados do SIS (2021).

De forma que cada um desses cateteres é uma possível porta de acesso de microrganismos causadores de IPCS, seja laboratorial ou clínica, por meio intraluminal, extraluminal, manipulação do $h u b$ ou soluções contaminadas. Eventualmente hospitalizações prolongadas ou recorrentes aumentam essas possibilidades e podem ser responsáveis pelo aumento dos eventos de ICS. Segundo Villar e Sellares (2019), em sua pesquisa, há relação entre essa causa e a incidência 9,6 vezes maior de bacteremia em pacientes portadores de cateteres tunelizados e submetidos à hemodiálise no Hospital Universitário de Canárias, Espanha.

Sobre isso, a média de internação para inserção do cateter de longa permanência pelo Sistema Único de Saúde (SUS) foi de 2,1 dias, entre os anos de 2015 e 2019, contabilizando também as não-ocorrências de IPCS. Observou-se que a Região Centro-Oeste apresentou a maior média do período, superando a nacional em até 2,86 vezes no ano de 2016 (Brasil, 2020). A Região Nordeste, por sua vez, manteve-se estável e acumulou a menor média nos anos do levantamento, ver Tabela 1. 
Tabela 1: Média de permanência (em dias) em unidade hospitalar para realização de procedimento de implante de cateter de longa permanência semi ou totalmente implantável, por ano e por região.

\begin{tabular}{lllllll}
\hline Região & $\mathbf{2 0 1 5}$ & $\mathbf{2 0 1 6}$ & $\mathbf{2 0 1 7}$ & $\mathbf{2 0 1 8}$ & $\mathbf{2 0 1 9}$ & Média \\
\hline Brasil & 2,3 & 2,2 & 2,1 & 1,9 & 1,9 & 2,1 \\
Norte & 2,7 & 2,6 & 2,7 & 3,6 & 2,5 & 2,8 \\
Nordeste & 1,6 & 1,7 & 1,7 & 1,4 & 1,4 & 1,6 \\
Sudeste & 1,6 & 1,8 & 1,6 & 1,6 & 1,9 & 1,7 \\
Sul & 2,8 & 2,1 & 2,1 & 2,2 & 2,2 & 2,3 \\
Centro-Oeste & 6,1 & 6,3 & 5,8 & 3,8 & 1,8 & 4,8 \\
\hline
\end{tabular}

Fonte: Autores, com dados do SIS (2021).

Embora a ocorrência de IPCS não deva ser conectada apenas à duração da internação hospitalar, tão pouco deve o ser ao tipo de cateter utilizado, como mostra os achados de um estudo sul-coreano de 2020, cujo levantamento apresentou que nos Estados Unidos da América a ocorrência de ICS em dispositivos de veia central com inserção periférica, conhecido como PICC, é semelhante àquela relativa ao tradicional CVC, adverso ao que se observou na Coréia do Sul, onde àqueles relativos ao PICC tem bem menor frequência se comparado ao CVC (Park, Moon, Pai, \& Kim, 2020).

Ainda que não possa ser taxada como causa desse resultado supracitado, efetivamente, a lavagem inadequada das mãos é responsável por parte das ocorrências. Tendo em vista que ao ser implementada de forma adequada à educação continuada e treinamento de profissionais de enfermagem foi responsável por uma redução de até 47,6\% da incidência de IPCS em Unidades de Terapia Intensiva Neonatal (UTIN) na Índia, conforme a pesquisa qualitativa conduzida entre os meses de janeiro de 2017 a dezembro de 2018, e que comparou os dados pré e pós intervenção em UTIN (Mohapatra et al., 2020).

Em vista disso uma ferramenta que atende bem esse propósito seria o bundle (pacote), forma estruturada de melhoria de processo, desenvolvido pelo Institute of Healthcare Improvement (IHI). Este método difere das listas e check-list, por tratarse de um conjunto de três a cinco elementos indispensáveis para realização de um procedimento, que devem ser objetivos, específicos para cada finalidade, baseados em evidências e cumpridos integralmente por um profissional ou equipe supervisionada por um responsável apenas (IHI, 2021). Segundo Manzo (2018), o bundle contribui de forma considerável para a redução das ICS, garantindo a qualidade e segurança da assistência.

Baseado na campanha "Protecting 5 Millions Lives" da IHI de 2006, a ANVISA instituiu um pacote com o objetivo de avaliar as ações e intervenções assistenciais, servindo de indicador de processo na prevenção das IPCS. Este é constituído de 5 elementos: higiene das mãos; precauções máximas de barreira na passagem do cateter; antissepsia com clorexidina; escolha do sítio de inserção adequado, com preferência para a veia subclávia nos casos de cateteres não tunelizados e reavaliação diária da necessidade de manutenção do cateter, com pronta remoção daqueles desnecessários. A adesão bem-sucedida dá-se no cumprimento bundle em sua integralidade para que ocorra melhorias substanciais (ANVISA, 2013).

Atendendo às recomendações da OMS, a ANVISA, responsável pelas ações nacionais de controle e prevenção de IRAS desde 1999, criou o Programa Nacional de Prevenção e Controle de Infecções Relacionadas à Assistência à Saúde (PNPCIRAS) alcançando bons resultados entre 2013 a 2015 na primeira versão do programa, em destaque a redução das densidades de incidência de IPCS, conforme Tabela 2. Já na segunda versão, de 2016 a 2020, a meta principal foi reduzir a incidência de IRAS em serviços de saúde, resultando em aumento da adesão e regularidade de notificação desse serviço (ANVISA, 2021). 
Tabela 2 - Dados de Notificação de Infecções Primárias de Corrente Sanguínea com Confirmação Laboratorial (IPCSL) em Unidades de Terapia Intensiva.

Número de Serviços que Notificaram por Ano - Total e de $\mathbf{1 0}$ a 12 meses

\begin{tabular}{|c|c|c|c|c|c|c|c|c|c|c|}
\hline & \multirow[b]{2}{*}{ TOTAL } & \multicolumn{2}{|c|}{2015} & \multirow{2}{*}{$\begin{array}{r}2016 \\
10 \text { A } 12\end{array}$} & \multirow[b]{2}{*}{ TOTAL } & \multirow{2}{*}{$\begin{array}{l}2017 \\
10 \text { A } 12\end{array}$} & \multirow[b]{2}{*}{ TOTAL } & \multirow{2}{*}{$\begin{array}{l}018 \\
10 \text { A } 12\end{array}$} & \multicolumn{2}{|c|}{2019} \\
\hline & & $10 \mathrm{~A} 12$ & TOTAL & & & & & & TOTAL & 10 A 12 \\
\hline \multicolumn{11}{|c|}{ UTI ADULTO } \\
\hline Brasil & 1349 & 1041 & 1429 & 1203 & 1518 & 1340 & 1598 & 1361 & 1636 & 1430 \\
\hline $\mathbf{N}$ & 69 & 45 & 72 & 50 & 76 & 61 & 92 & 67 & 99 & 82 \\
\hline NE & 186 & 74 & 234 & 189 & 255 & 223 & 292 & 202 & 298 & 234 \\
\hline $\mathrm{CO}$ & 131 & 107 & 134 & 98 & 148 & 123 & 149 & 124 & 153 & 132 \\
\hline SE & 755 & 633 & 765 & 679 & 802 & 734 & 827 & 751 & 842 & 768 \\
\hline $\mathbf{S}$ & 208 & 182 & 224 & 187 & 237 & 199 & 238 & 217 & 244 & 214 \\
\hline \multicolumn{11}{|c|}{ UTI NEONATAL } \\
\hline Brasil & 576 & 435 & 608 & 531 & 627 & 556 & 672 & 592 & 750 & 664 \\
\hline $\mathbf{N}$ & 35 & 25 & 36 & 25 & 37 & 35 & 48 & 37 & 50 & 42 \\
\hline NE & 64 & 24 & 79 & 65 & 96 & 77 & 105 & 77 & 118 & 96 \\
\hline $\mathrm{CO}$ & 43 & 31 & 39 & 35 & 45 & 34 & 52 & 44 & 54 & 50 \\
\hline SE & 371 & 309 & 386 & 350 & 381 & 349 & 393 & 369 & 399 & 362 \\
\hline $\mathbf{S}$ & 64 & 40 & 68 & 56 & 71 & 630 & 74 & 65 & 129 & 114 \\
\hline \multicolumn{11}{|c|}{ UTI PEDIÁTRICA } \\
\hline Brasil & 430 & 319 & 455 & 373 & 488 & 416 & 525 & 440 & 571 & 497 \\
\hline $\mathbf{N}$ & 36 & 19 & 32 & 24 & 34 & 30 & 38 & 28 & 42 & 37 \\
\hline NE & 50 & 19 & 61 & 43 & 64 & 57 & 77 & 58 & 95 & 67 \\
\hline $\mathrm{CO}$ & 31 & 23 & 29 & 20 & 34 & 24 & 38 & 28 & 41 & 32 \\
\hline SE & 261 & 221 & 280 & 241 & 292 & 258 & 303 & 261 & 321 & 300 \\
\hline $\mathbf{S}$ & 54 & 37 & 53 & 45 & 64 & 47 & 69 & 57 & 72 & 61 \\
\hline
\end{tabular}

Fonte: Autores, com dados do SIS (2021).

Observa-se, na Tabela 2, que o Brasil realizou um número crescente de notificações de IPCSL, no período de 2015 a 
2019. Com uma média de 1506 eventos totais por ano, a UTI adulto correspondeu ao maior número, já na UTI neonatal e pediátrica a média foi de 646 e 493, respectivamente. No entanto, segundo o número de serviços de saúde que realizaram notificações existe uma disparidade na quantidade total entre as regiões do país, haja vista que o Sudeste é a região que mais notifica, enquanto a Região Norte é a que menos a realiza.

Nota-se ainda, que a quantidade de serviços que realizou notificações totais foi consideravelmente maior que aqueles que notificaram de dez a doze meses. No Brasil, a razão entre essas duas variáveis, em 2019, por exemplo, foi de 0,87, ver Quadro 1. Essa relação se apresentou menos favorável na Região Nordeste, em que no mesmo ano foi de 0,78, e mais proporcional na Região Sudeste, que alcançou o valor de 0,91.

Quadro 1: Cálculo da razão da frequência de notificação de IPCSL em UTI no Brasil.

Razão da Notificação por Frequência da Notificação = Número de Serviços que Notificaram por Ano de 10 a 12 meses

Número de Serviços que Notificaram por Ano Total

Fonte: Autores (2021).

Percebe-se que a fidedignidade das informações referente à incidência de IPCSL ainda é insatisfatória em virtude dessa subnotificação, além disso, a vigilância de eventos adversos ganhou força apenas na última década, no Brasil, e a realização das notificações através de formulário eletrônico encaminhados à ANVISA, formSUS, precisa ser incorporado de forma substancial na rotina dos serviços de saúde (Maia, Freitas, Gallo, \& Araújo, 2018).

Portanto, após a análise e discussão do levantamento de dados supracitados classificados por Região, observa-se na tabela 3 o quantitativo da densidade de incidência de Infecção Primária de Corrente Sanguínea Laboratorial Relacionada ao uso de Cateter Venoso Central em pacientes sob cuidados intensivos, organizada por Unidade de Terapia Intensiva, de acordo com faixa etária. O número de IPCSL é calculado a cada 1.000 CVC-dia, sendo a UTI adulto, pediátrica e neonatal, no período de 2015 a 2019.

Tabela 3 - Densidade de incidência de Infecção Primária de Corrente Sanguínea Laboratorial Relacionada ao uso de Cateter Venoso Central em Pacientes Internados em Unidades de Terapia Intensiva, no período de 2015 a 2019, no Brasil.

\begin{tabular}{lccccc}
\hline Tipo de UTI & $\mathbf{2 0 1 5}$ & $\mathbf{2 0 1 6}$ & $\mathbf{2 0 1 7}$ & $\mathbf{2 0 1 8}$ & $\mathbf{2 0 1 9}$ \\
\hline UTI adulto & 4,81 & 4,67 & 4,44 & 4,15 & 3,92 \\
UTI pediátrica & 5,73 & 5,28 & 4,86 & 4,62 & 4,38 \\
UTI neonatal & 7,84 & 7,81 & 6,82 & 7,47 & 7,85 \\
\hline
\end{tabular}

Fonte: Autores, com os resultados do estudo (2021).

Em epidemiologia, a densidade de incidência trata-se do quantitativo da frequência com que surge determinado evento de interesse do pesquisador, no decorrer de um tempo estabelecido, referente ao tamanho de uma população. Assim, é possível avaliar a incidência de casos novos de um problema de saúde ou doença em um recorte de tempo, sendo possível estabelecer metas e projetar resultados para lidar com o problema (Merchan-Hamann, Tauil, \& Costa, 2000).

Logo, constata-se, segundo os dados da tabela 3, que a densidade de incidência de IPCSL, em UTI adulto, caiu 0,89 em 2019 em comparação a 2015, ou seja, no último ano a cada 1.000 CVC-dia a quantidade de infecções foi de 3,92. Ademais, a média no período investigado foi de 4,39. Nota-se, também, uma redução de 1,35 em UTI pediátrica, visto que de 2015 a 2019 
houve uma queda anual do quantitativo de infecções e a média foi de 4,97. Por outro lado, houve um aumento no número em UTI neonatal de 0,1, mantida a média de 7,55 infecções.

À vista disso, as IPCSL ainda persistem em um número significativo no Brasil, evidenciadas pelas altas taxas de densidade de incidência, as quais podem ter causas multifatoriais e a educação permanente da equipe de saúde, sobre os protocolos para prevenção, é a base para a redução desses eventos. Isto posto, um estudo transversal em um hospital de Belo Horizonte, em que avaliou o conhecimento da equipe de enfermagem e dos médicos com relação às principais medidas de prevenção da infecção de corrente sanguínea, no cuidado ao CVC, desde a inserção, como durante o seu manuseio, mostrou que o conhecimento da equipe de enfermagem foi abaixo de 50\% (Silva \& Oliveira, 2018).

Sabe-se que o conhecimento referente às medidas preventivas é imprescindível para minimizar as IRAS e deve ser compartilhado e aplicado através de capacitação da equipe e educação continuada em saúde, com vistas a melhoria das práticas assistenciais exercidas. Outro estudo, também na capital de Minas Gerais, realizado em três unidades de terapia intensiva, concluiu que a equipe de saúde apresentou fragilidades importantes frente ao conhecimento necessário para prevenir as IPCS associada ao CVC e a adesão aos protocolos. Reforçou ainda, a necessidade de incentivo à implementação de ações e estratégias que contribuam para uma prática assistencial pautada na segurança do paciente (Costa et al., 2020).

Salienta-se que, as responsabilidades pelos agravos aos pacientes advindos da assistência à saúde, como a infecção de corrente sanguínea debatida neste estudo, não devem ser apenas taxadas como consequência de fragilidades no que se refere às capacitações realizadas pelos serviços de saúde. Consequentemente, a educação em saúde não exime a busca pelo conhecimento individual pelos profissionais, que deve ser mantida após a formação acadêmica, uma vez que o conhecimento na área está em constante mudança, principalmente, passível a diversas atualizações, de forma a atingir melhorias nos cuidados prestados.

Destaca-se que os fatores de risco em Unidades Terapia Intensiva Neonatal diferenciam-se por características próprias dessa faixa etária como: o peso abaixo do normal ao nascer, recém-nascidos pré-termo e o tempo de permanência do CVC, pois contribuem para o aumento do risco de aquisição da IPCS nesta unidade, evidenciado na tabela 3 no recorte temporal estabelecido. Contudo, existem poucos estudos nas bases nacionais voltados para a população neonatal (Rosado et al., 2018).

Todos esses fatores associados discutidos, como também os que de alguma forma não foram abordados, contribuem para a manutenção do número considerável de infecções, devido ao amplo alcance que a densidade de incidência de IPCSL tem, no que diz respeito à segurança do paciente. Os quais refletem a necessidade de avaliação e monitoramento dos programas e estratégias vigentes de prevenção das IRAS, que visam minimizar essas ocorrências no país.

\section{Conclusões}

Em síntese, é imprescindível ressaltar a importância clínica do cateter central no tratamento do paciente, uma vez que possibilita a infusão de variadas soluções, como, grandes volumes de expansores plasmáticos, sangue e hemoderivados, sem comprometer a rede venosa periférica. Principalmente em pacientes que se encontram hemodinamicamente instáveis e necessitam de drogas vasoativas para manutenção dos parâmetros vitais, para sua monitorização, e ainda, nutrição parenteral e realização de hemodiálise.

Portanto, após análise dos dados levantados, observou-se uma redução na média de dias de internação para realizar o procedimento de inserção do CVC e, simultaneamente, na densidade da incidência de IPCSL tanto na UTI adulto quanto na pediátrica, excetuando-se a neonatal, o que requer uma investigação à parte acerca de suas causas. Concomitantemente, ocorreu um aumento progressivo no número de serviços de saúde que realizaram notificação dessa categoria de evento, porém ainda é necessária a realização de intervenções para adesão dessa prática, exaltando o prejuízo da carência desses dados.

Percebe-se que, com relação às notificações, há uma incongruência na emissão desses dados, tanto na frequência quanto na quantidade de eventos, evidenciada pela diferença entre o quantitativo total de serviços de saúde que realizaram notificações, 
independentemente da constância mensal, e aqueles que o fizeram durante 10 meses ou mais ao ano. É indubitável a importância do registro dessas infecções, pois a quantidade de dados gerados, se satisfatória, pode tornar a informação consistente e com uma ampla representatividade. Pondera-se que esses incidentes significam muito quando considerado a segurança do paciente, além de serem causas do aumento de tempo de internação e elevação significativa dos custos relacionados ao tratamento.

Nessa perspectiva, o conhecimento da real magnitude dessas incidências, a constância de registros, a educação continuada, a atualização profissional individual e adesão massiva ao sistema de notificação tornam-se indispensáveis para que medidas de prevenção e promoção à saúde sejam criadas, bem como a ampliação de conhecimento sobre o tema, contribuindo para criação de culturas institucionais, mudança nas práticas assistenciais, tecnológicas e regulamentações para assegurar a redução de danos, aumentando a segurança dos pacientes.

Dessa forma, sugerem-se os fatores relacionados às IPCS para cada faixa etária do estudo como direção para futuras pesquisas, com vistas a conhecer e delimitar medidas de segurança mais adequadas, como também, pesquisas que avaliem e levantem dados sobre as próprias notificações nas Unidades de Terapia Intensiva. Assim, permite-se perceber as causas da divergência entre as frequências de notificações encontradas neste estudo e, para melhoria da confiabilidade, consolidar os fatores de uniformização desses dados em todo território nacional.

\section{Referências}

ANVISA (2019). Boletim Segurança do Paciente e Qualidade em Serviços de Saúde no Brasil n²2. Avaliação dos Indicadores Nacionais de Infecções Relacionadas à Assistência à Saúde (IRAS) e Resistência Microbiana (RM), 2019. https://app.powerbi.com/view?r=eyJrIjoiZjQ5ZDhjZmEtNDdhO C00MDk3LWFiNDEtN zg0MmE4MmE2MjlhIiwidCI6ImI2N2FmMjNmLWMzZjMtNGQzNS04MGM3LWI3MDg1ZjV1ZGQ4MSJ9\&pageName=Repor tSectionac5c0437dbe709793b4b.

ANVISA (2013). Critérios Diagnósticos de Infecções Relacionadas à Assistência à Saúde. http://bvsms.saude.gov.br/bvs/publicacoes/criterios_ diagnosticos_infeccoes_assistencia_saude.pdf.

ANVISA (2021). Programa Nacional de Prevenção e Controle de Infecções Relacionadas à Assistência à Saúde (PNPCIRAS) 2021 a 2025. Brasília. https://www.gov.br/anvisa/pt-br/centraisdeconteudo/publicacoes/servicosdesaude/publicacoes/pnpciras_2021_2025.pdf.

Brasil (2020). Ministério da Saúde. Sistema de informações em Saúde - SIS. Banco de Dados do Sistema Único de Saúde - DATASUS. http://www2.datasus.gov.br/DATASUS/index.php?area=02.

Brasil (2014). Ministério da Saúde. Documento de Referência para o Programa Nacional de Segurança do Paciente. https://bvsms.saude.gov.br/bvs/publicacoes/documento_referencia_programa_nacional_seguranca.pdf.

Chaiyakulsil, C. \& Pharadornuwat, O. (2020) Can central venous access device care bundles and regular feedback reduce central line-associated complications in pediatric patients? Clin Exp Pediatr. 2021;64(3):123-129. https://www.e-cep.org/journal/view.php?doi=10.3345/cep.2020.00143.

Costa, C. A. B., Araújo, F. L., Costa, A. C. L., Côrreia, A. R., Kusahara, D. M. \& Manzo, B. F. (2020). Bundle de Cateter Ven oso Central: conhecimento e comportamento de profissionais em Unidades de Terapia Intensiva adulto. Revista da Escola de Enfermagem da USP [online]. V. 54, e03629. https://www.scielo.br/j/reeusp/a/CW7dqY3H6YYnrQ8L3rjPHLN/?lang=pt.

Fortunatti, C. F. P. (2017). Impact of two bundles on central catheter-related bloodstream infection in critically ill patients. Revista Latino-Americana de Enfermagem [online], 25. https://doi.org/10.1590/1518-8345.2190.2951.

GIL, A. C (1999). Métodos e técnicas de pesquisa social. (5a ed.), Atlas.

Karapanau, A., Vieru, A. M., Sampanis, M. A., Pantazatou, A., Deliolanis, I., Daikos, G. L. \& Samarkos, M. (2020). Failure of central venous catheter insertion and care bundles in a high central line-associated bloodstream infection rate, high bed occupancy hospital. American Journal of Infection Control, 48(7):770776.https://europepmc.org/article/med/31911066.

Llapa-Rodríguez, E. O., Oliveira, J. K. A., Melo, F. C., Silva, G. G., Mattos, M. C. T. \& Júnior, V. P. M (2019). Insertion of central vascular catheter: adherence to infection prevention bundle. Revista Brasileira de Enfermagem [online], 72(3). https://doi.org/10.1590/0034-7167-2018-0124.

Maia, C. S., Freitas, D. R. C., Gallo, L. G. \& Araújo, W. N. (2018). Notificações de eventos adversos relacionados com a assistência à saúde que levaram a óbitos no Brasil, 2014-2016. Epidemiologia e Serviços de Saúde [online], 27(2), e2017320. https://www.scielo.br/j/ress/a/6 7kfbVWmYrCNSyZ5NmyXpjR/?lang=pt.

Manzo, B. F., Corrêa, A. R., Vieira, C. P. V., Mota, R. L., Oliveira, J., Simão, D. A. \& Guimarães, G. L. (2018). Bundle de cateter central: comportamento de profissionais da saúde em neonatologia. Revista Brasileira de Enfermagem [online], 12(1). https://periodicos.ufpe.br/revistas/revi staenfermagem/article/view/23236/25841. 
Research, Society and Development, v. 10, n. 10, e565101019150, 2021

(CC BY 4.0) | ISSN 2525-3409 | DOI: http://dx.doi.org/10.33448/rsd-v10i10.19150

professionals about bundled strategies of central venous catheter. Revista Brasileira de Enfermagem [online], 72(1). https://doi.org/10.1590/0034-7167-20180164

Merchan-Hamann, E., Tauil, P. L. \& Costa, M. P.(2000). Terminologia das medidas e indicadores em epidemiologia: subsídios para uma possível padronização da nomenclatura. Inf. Epidemiol. Sus, 9(4),. 276-284. http://scielo.iec.gov.br/scielo.php?script=sci_arttext\&pid=S0104-16732000000400006\&lng=pt\&nrm=iso.

Mohapatra, S., Kapil, A., Suri, A., Pandia, M. P., Bhatia, R., Borkar, S., Dube, S. K., Jagdevan, A., George, S., Varghese, B. \& Dabral, J. (2020). Impact of Continuous Education and Training in Reduction of Central Line-associated Bloodstream Infection in Neurointensive Care Unit. Indian J Crit Care Med.; 24(6): 414-417. https://www.ncbi.nlm.nih.gov/pmc/articles/PMC7435091/.

Park, S., Moon, S., Pai, H. \& Kim, B. (2020). Appropriate duration of peripherally inserted central catheter maintenance to prevent central line-associated bloodstream infection. PLOS ONE 15(6): e0234966. https://journals.plos.org/plosone/article?id=10.1371/journal.pone.0234966.

Richardson, R. J. (1999). Pesquisa social: métodos e técnicas. (3a ed.), Atlas.

Rosado, V., Camargos, P. A. M., Anchieta, L. M., Bouzada, M. C. F., Oliveira, G. M., Clemente, W. T. \& Romanelli, R. M. C. (2018). Risk factors for central venous catheter-related infections in a neonatal population - systematic review. Jornal de Pediatria [online], 94, 3-14. https://www.sciencedirect.com/science/article/pii/S0021755717307829?via\%3Dihub.

Silva, A. G. \& Oliveira, A. C. (2018). Conhecimento autorreferido das equipes médica e de enfermagem quanto às medidas de prevenção de infecção da corrente sanguínea. Texto \& Contexto - Enfermagem [online], 27(3). https://www.scielo.br/j/tce/a/wsqsTSj6Q9pgfWCpfH7JQ6S/?lang=pt.

Silva, A. G. \& Oliveira, A. C. (2018). Impacto da implementação dos bundles na redução das infecções da corrente sanguínea: Uma Revisão Integrativa. Texto \& Contexto - Enfermagem [online], 27(1). https://doi.org/10.1590/0104-07072018003540016.

Villar, I. S. \& Sellares, V. L. (2019). ¿Se infectan más los catéteres tunelizados para hemodiálisis cuando los pacientes ingresan en el hospital? Enfermería Nefrológica, 22(3).. https://enfermerianefrologica.com/revista/article/view/4083. 